\title{
BEHAVIOUR OF SWEEP LINE IN PAIR-TRAWLING*
}

\author{
Otohiko SUzukI**
}

(Received July 17, 1962)

In the previous papers ${ }^{1,21}$ differential equations about the form and tension of sweep line have been treated and the experimental data for the case of a uniform nylon twine have been analyzed in terms of the theory presented.

In pair-trawling, it has been pointed out ${ }^{3)}$ that the sweep line, in earlier stages of towing, shows two different kinds of behaviour. The first pattern, which appears in the process from the layout of rope to the stage that the deformation of rope propagates to the wing tip of net by towing, can be reduced to the case treated previously. ${ }^{1) 2}$ In the second process, the inclination angle of a rope to the towing direction at wing tip varies as a function of the towed distance.

In the present work on the sweep line of pair-trawl, accordingly, the focal point on this study is in the second process. As an extension of the previous theory, ${ }^{11}$ the behaviour of sweep line in the process will be discussed on the assumption that the resistance of net is constant thruoghout the course of towing.

\section{Theoretical Consideration}

According to one of the most popular patterns of layout in pair-trawling, a uniform rope is laid, on a horizontal plane of sand, in such a form that it makes three sides $\widehat{\mathrm{DA}}, \overline{\mathrm{AB}}$ and $\overline{\mathrm{BC}}$ of a rectangle (Fig. 1). Here, assume that a net is attached to the rope at the mid-point $O$ of the side $\bar{A} \bar{B}$. If both ends $\mathrm{C}$ and $\mathrm{D}$ of the rope is pulled to a definite direction $\overline{\mathrm{BC}}$ and $\overline{\mathrm{AD}}$, the pattern assumed by both sides of rope will change symmetrically with respect to the central line. At a certain instant of towing process, the deformation of rope will propagate to the point $O$. The behaviour of rope in this course has been discussed previously. ${ }^{1,2)}$ In the subsequent process of towing, the

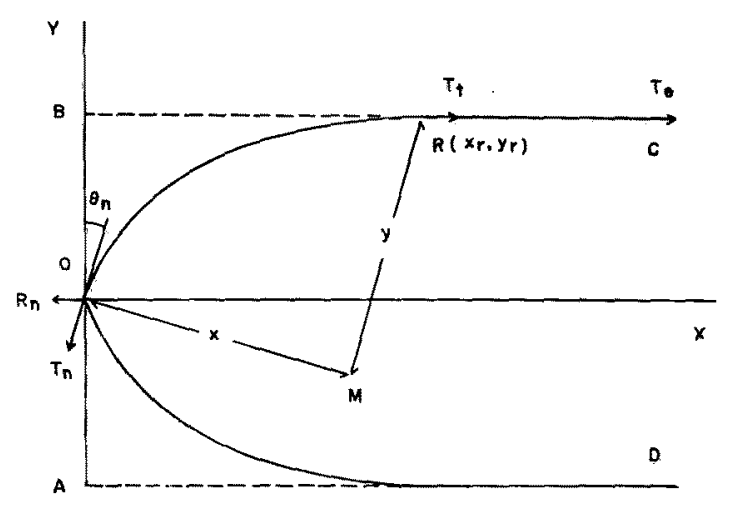

Fig. 1. Form of sweep line in pair-trawling. rope will be bent at the point $O$, owing to the resistance of net. As the rope is towed on further, an included angle made by the both sides of rope at the point $\mathrm{O}$

* Studies on the kinematic behaviour of the ground rope of the trawl net.V.

** Department of Fisheries, Kyoto University, Maizuru (鈴木乙彦, 京大・水産) 
will decrease, and the net starts to move when the towing component of tension on the rope at the point $O$ exceeds the resistance of net.

Now, referring to Fig. 1, take the $y$-axis to the direction of the part $\overline{\mathrm{AB}}$ of rope originally laid, and choose the origin at the mid-point $O$ of the originally laid rope. We may confine our discussion to the left half of the curve by consideration of symmetry. Denote by $T_{n}$ the tension acting on the rope at the point $O, \theta_{n}$ the angle of inclination of $T_{n}$ to the positive direction of the $y$-axis. Then, $T_{n}$ be the tension acting on the rope at the point $\mathrm{R}$, at which the curve comes in contact with the line $\overline{\mathrm{BC}}$ as shown in Fig. 1; here, from the initial condition that $\overline{\mathrm{OB}}$ is perpendicular to $\overline{\mathrm{BC}}$, the inclination angle of $T_{t}$ to the positive $y$-axis keeps $\pi / 2$. The same mechanical relation as discussed in the preceding papers ${ }^{1,2}$, should hold also in this case. Hence, we have the relation between the tensions, $T_{n}$ and $T_{t}$, acting on the rope at the respective points, $O$ and $R$,

$$
\frac{T_{t}}{T_{n}}=e^{\kappa\left(\pi / 2-\theta_{n}\right)}
$$

where $\kappa=K_{t} / K_{n}$, denoting by $K_{t}$ and $K_{n}$ the tangential and the normal component of frictional force per unit length of rope, respectively. Moreover, the arc length $s_{r}$ (= $\overparen{\mathrm{OR}}$ ) of the curve is given by

$$
s_{r}=\frac{T_{n}}{K_{n}} \frac{1}{\kappa}\left(e^{\left.\kappa i \pi / 2-\theta_{n}\right)}-1\right) .
$$

In order to obtain the coordinates of the point $\mathrm{R}$, some attentions should be paid. In the reference ${ }^{1)}$ cited above, we have defined the $y$-axis of the rectangular coordinates system to the direction of tension acting on rope at the origin. As a consequence, the values of $x$ and $y$, expressed by the equations (5) and (6) of the reference ${ }^{13}$, give the lengths in direction of $\overline{\mathrm{OM}}$ and $\overline{\mathrm{MR}}$, respectively, as in Fig. 1. The present coordinate axes can be obtained by rotating the above mentioned coordinate system about the origin to the positive sense as far as the angle $\theta_{n}$. Therefore, the projections, $x_{r}$ and $y_{r}$, of $s_{r}$ to the present coordinate axes become

$$
\begin{aligned}
& x_{r}=x \cos \theta_{n}+y \sin \theta_{n}, \\
& y_{r}=y \cos \theta_{n}-x \sin \theta_{n} .
\end{aligned}
$$

Substitution of $x$ and $y$, given from (5) and (6) of the reference ${ }^{1)}$, into the above expressions gives:

$$
\begin{aligned}
& x_{r}=\frac{T_{n}}{K_{n}} \frac{1}{\kappa^{2}+1}\left[e^{\kappa\left(\pi / 2-\theta_{n}\right)} \kappa-\left(\kappa \sin \theta_{n}-\cos \theta_{n}\right)\right], \\
& y_{r}=\frac{T_{n}}{K_{n}} \frac{1}{\kappa^{2}+1}\left[e^{\kappa\left(\pi / 2-\theta_{n}\right)}-\left(\kappa \cos \theta_{n}+\sin \theta_{n}\right)\right] .
\end{aligned}
$$

The equation (4) is rewritten to a dimensionless form, 


$$
\frac{K_{n}}{T_{n}} y_{r}=\frac{1}{\kappa^{2}+1}\left[e^{\kappa\left(\pi / 2-\theta_{n}\right)}-\left(\kappa \cos \theta_{n}+\sin \theta_{n}\right)\right]
$$

The right-hand member of the above equation is a function of $\theta_{n}$, for the given value of $x$. Then, if the value of $y_{r}(=\overline{\mathrm{OB}})$ is determined according to the experimental condition, the values of $K_{n} / T_{n}$ and thus in turn $T_{n}$ can be obtained as functions of $\theta_{n}$. Moreover, Fig. 1 gives geometrically the towed distance $l$ of the rope end:

$$
l=x_{r}+y_{r}-s_{r} .
$$

The equation (6) gives $l$ as a function of $\theta_{n}$, and in reverse the towing component $T_{n} \sin \theta_{n}$ acting at the wing tip can also be given as a function of $l$. Here, denoting by $R_{n}$ the resistance of net in motion, we have, as the condition under which the net starts to move

$$
\frac{R_{n}}{2}=T_{r_{0}}^{*} \sin \theta_{n}^{*},
$$

where $T_{n}{ }^{*}$ and $\theta_{n}{ }^{*}$ are the tension of rope at the point $\mathrm{O}$ and its inclination angle, respectively, when the net starts to move. If the value of $R_{n}$ is known, the values of $\theta_{n}{ }^{*}$ and $T_{n}{ }^{*}$ are determined in terms of the equation (7). It is seen theoretically that the rope will be towed keeping the same stationary pattern as assumed when the net starts to move, if $R_{n}$ keeps a constant value in the whole course of towing.

\section{Experimental}

The outline of experimental apparatus has been illustrated previously.4 A uniform flexible twin of $3 \mathrm{~mm}$. in diameter was laid at first, on a horizontal plane of dry sand, in such a form as shown in Fig. 1, letting $\overline{\mathrm{AD}}=\overline{\mathrm{BC}}=120 \mathrm{~cm}$. and $\overline{\mathrm{AO}}=\overline{\mathrm{OB}}=30 \mathrm{~cm}$. A small lead ball of weight $1.70 \mathrm{gr}$. was attached to the twine at the point $\mathrm{O}$, in place of the net. In order to keep the distance between the both ends $C$ and $D$ of twine to be constant, a thin bamboo rod of definite length $60 \mathrm{~cm}$. was connected to each end of the twine. Thus, the both ends were pulled simultaneously at speeds in the range of 1.25 to $1.66 \mathrm{~cm} / \mathrm{sec}$. In the first experiment, the towing force was measured at a regular interval $5 \mathrm{~cm}$. of dragged distance, and then the form assumed by the twine was photographed from the top. Here, the bamboo rod has no influence upon the measured towing force, as the rod is kept afloat off the sand surface in motion by the taut hauling thread. In the second experiment, the measurements were made at a regular interval $1 \mathrm{~cm}$. of towed distance between 14 and $21 \mathrm{~cm}$., which was presumed to be the range to be treated in the present study. In the experiment, the towing force was measured rather larger than its intrinsic value, owing to the slight contact of bamboo rod with the sand surface.

\section{Results and Discussion}

According to the preceding experiment, ${ }^{2)}$ the values of $K_{t}$ and $K_{n}$ of the twine 
used have been known to be $0.0440 \mathrm{gr} . / \mathrm{cm}$. and $0.0517 \mathrm{gr} . / \mathrm{cm}$., respectively. Substitution of the value $\kappa=0.8500$, together with $y_{r}(=\overline{\mathrm{OB}})=30 \mathrm{~cm}$. derived from the experimental condition, into the equation (5) gives the relationship between $\theta_{n}$ and $T_{n}$, as shown in Fig. 2. On the other hand, the frictional resistance $R_{n}$ of the lead ball is determined independently and is found to be $2.1100 \mathrm{gr}$, , then we get $\theta_{n}{ }^{*}=30^{\circ}$, by combining the equation (7) and the result given in Fig. 2.

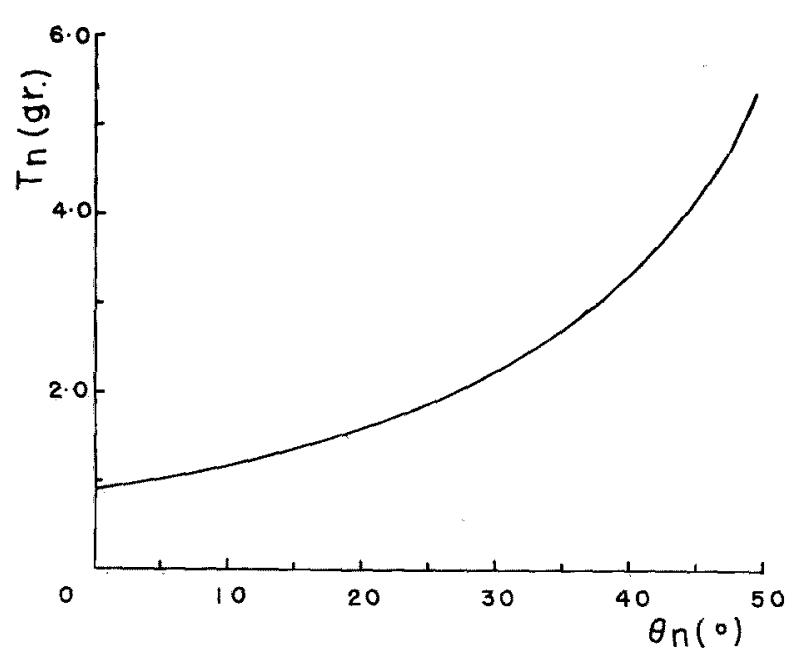

Fig. 2. Tension $T_{n}$ plotted as a function of $\theta_{n}$.

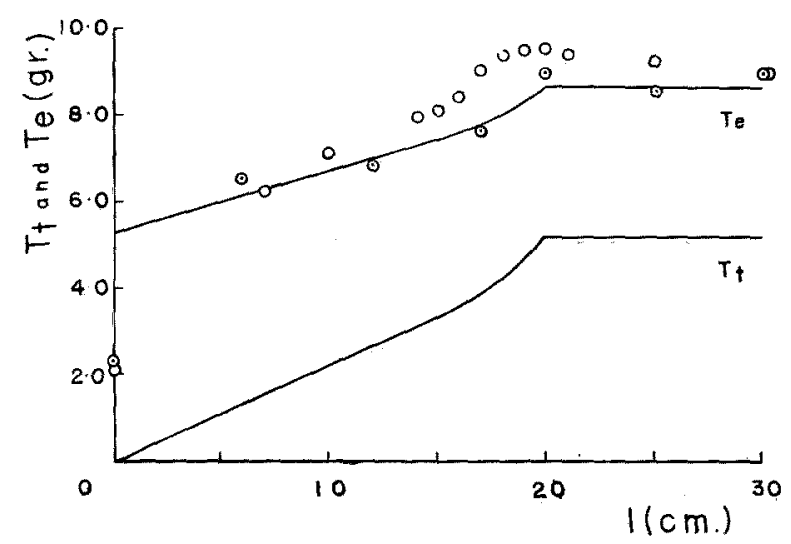

Fig. 3. Calculated and observed values of tension $T_{e}$ plotted against towed distance $l$. The marks $\odot$ and show duplicate experiments.
Substituting $\theta_{n}=0^{\circ}$ and $T_{n}$ $=0.9070$ gr., given from Fig. 2 , to the equations (2), (3) and (4), the towed distance when the deformation of rope propagates to the point $O$ is determined to be $15.34 \mathrm{~cm}$. by means of the equation (6). Similarly, the towed distance when the ball starts to move is determined to be $20.06 \mathrm{~cm}$. In the region $l=0 \sim 15.34 \mathrm{~cm}$., the data about the form and the tension of the twine treated have already been obtained by the previous experiment, ${ }^{2)}$ accordingly, the data may be used in the present study. Now we proceed to our calculation in the range $l=15.34$ $\sim 20.06 \mathrm{~cm}$, in which the angle $\theta_{n}$ varies with $l$. Since the relation between $\theta_{70}$ and $T_{70}$ has been given and, moreover, $l$ can be expressed in terms of $\theta_{n}$, we can get the relation between $l$ and $T_{r}$. Then the tension $T_{t}$ acting on the twine at the point $R$ can be expressed as a function of $l$. From the experimental condition $\overline{\mathrm{BC}}=120 \mathrm{~cm}$., the tangential frictional force $K_{t} \times(\overline{\mathrm{RC}})$ acting on the linear part $\overline{\mathrm{RC}}$ of rope is calculated as a function of $l$, and thus the tension $T_{e}\left[=T_{t}+K_{t} \times(\overline{\mathrm{RC}})\right]$ at the end of twine is also determined as a function of $l$. In Fig. 3 the calculated and observed values of $T_{e}$, including the calculated value of $T_{t}$, are plotted against $l$, the both values of $T_{e}$ agree with each other within experimental 
error. The characteristic values, $s_{r}, \quad x_{r}$ and $y_{r}$, of the pattern assumed by the twine can also be obtained as functions of $l$. In Fig. 4 are shown these relations, including the dragged distance $d$ of the ball, together with their experimental ones. They agree satisfactorily with each other.

The author is indebted to Prof. T. Kawaram for his valuable advice and revision.

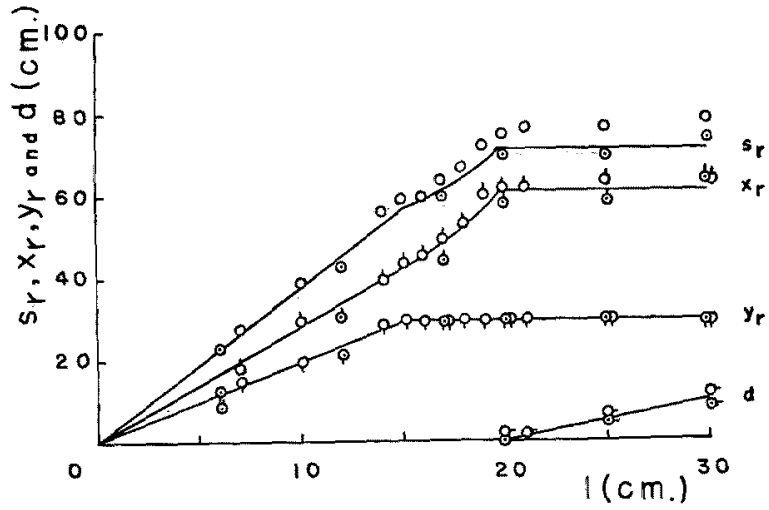

Fig. 4. Calculated and observed values of $s_{r}, x_{r}, y_{r}$ and $d$ plotted against $l$.

\section{References}

1) SUzukI, O.: Bull. Jap. Soc. Sci. Fish., 28 985-987 (1962).

2) SUzUKI, O.: ibid., 28 988-991 (1962).

3) SUzuKI, O. and T. KaWAKaMI: ibid., 26 204-206 (1960).

4) KaWAKaMI, T. and O. SUZUKI: ibid., 25 413-416 (1959). 